\title{
Käfer ist Vorbild für strahlend weiße Oberflächen
}

\author{
Ein Käfer inspirierte Wissenschaftler zur Entwicklung eines neuen Verfahrens, mithilfe dessen \\ Oberflächen strahlend weiß werden. Die weiße Optik wird dabei durch nanostrukturierte \\ Polymerfolie erreicht - ohne Einsatz von umweltbelastendem Titandioxid.
}

Anja Frisch

Polymerfolien, die extrem dünn sind und eine hohe Lichtstreuung aufweisen, sind das Ergebnis eines neuen Verfahrens aus dem Karlsruher Institut für Technologie (KIT). Das kostengünstige Material lässt sich industriell auf unterschiedlichste Gegenstände aufbringen, um ihnen eine attraktive weiße Optik zu verleihen. Zudem trägt das Verfahren dazu bei, dass Produkte umweltfreundlicher entwickelt werden können.

\section{Kostengünstige weiße Optik ohne Titandioxid}

Eine strahlend weiße Oberfläche lässt Möbel und andere Gegenstände sauber, hell und modern wirken. Bislang ist Titandioxid das Standardpigment, um Lacke, Farben und Kunststoffe, aber auch Kosmetika, Lebensmittel, Kaugummi oder Tabletten weiß zu färben. Das Pigment steht jedoch in der Kritik. Titandioxid hat einen sehr hohen Brechungsindex. Es reflektiert

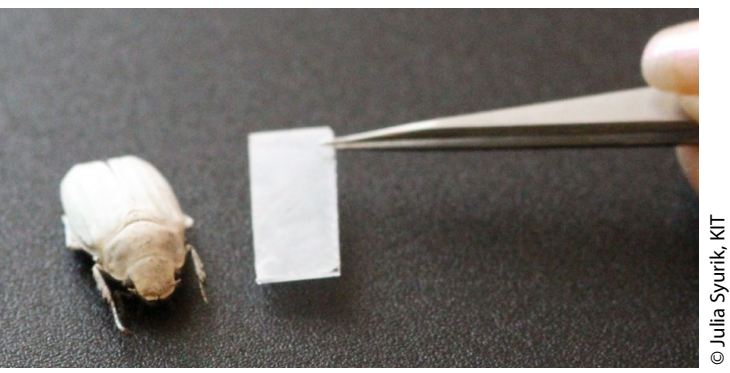

Nach dem Vorbild des weißen Käfers Cyphochilus insulanus erzeugt ein nanostrukturierter Polymerfilm eine strahlend weiße Beschichtung. einfallendes Licht fast vollständig. Es hat jedoch den Nachteil, dass sich seine Partikel nicht abbauen und dadurch auf Dauer die Umwelt belasten. Zudem gibt es Bedenken, dass Titandioxid möglicherweise gesundheitsschädlich sein könnte. Mit der neuen Technologie wird die Verwendung von umwelt- und gesundheitsschädlichen Pigmenten umgangen, indem poröse Polymerstrukturen mit vergleichbar hoher Streuung erzeugt werden. Inspiriert wurden die Forschenden von dem weißen Käfer Cyphochilus insulanus, dessen Schuppen dank einer speziellen Nanostruktur seines Chitinpanzers weiß erscheinen. Nach diesem Vorbild werden aus Polymeren feste, poröse Nanostrukturen hergestellt, die einem Schwamm ähneln. Wie die Bläschen von Rasier- oder Badeschaum sorgt auch hier die Struktur für eine Streuung des Lichts, die das Material weiß wirken lässt. Die neue Technik für eine kostengünstige und unbedenkliche weiße Optik eignet sich für verschiedenste Oberflächen.

\section{Umweltfreundliche Materialien nach dem Vorbild der Natur}

Die mit dem Verfahren gefertigten Polys merfolien sind extrem dünn, flexibel und leicht, aber dennoch mechanisch stabil und lassen sich industriell auf unterschiedliche Produkte aufbringen. Bei einer Stärke von $9 \mu \mathrm{m}$ - neun Tausendstel Millimeter reflektiert die neu entwickelte Polymerfolie mehr als $57 \%$ des einfallenden Lichts. 80 bis $90 \%$ sind bei einer dickeren Folie erreichbar. Für die Entwicklung wurde die schwammförmige Mikrostruktur auf Acrylglas aufgebracht. Das Verfahren lässt sich jedoch auf viele andere Polymere übertragen. Neben Folien lassen sich auch ganze Gegenstände entsprechend weiß färben. Als nächster Schritt sind Partikel geplant, zum Beispiel kleine Kügelchen, um sie in andere Materialien einbringen zu können. So gibt es bereits Anfragen von Unternehmen, die bestrebt sind, ihre Produkte umweltfreundlicher zu machen. Während Ingenieurinnen und Ingenieure häufig Lösungen mit Materialien aus vielen verschiedenen chemischen Elementen entwickelten, beschränkt sich die Natur meist auf ein einziges Grundmaterial, das dank einer komplexen dreidimensionalen Struktur interessante mechanische, optische oder physikalisch-chemische Eigenschaften aufweist. Die Bionik, die sich damit beschäftigt, die Phänomene der Natur zu verstehen und $\mathrm{zu}$ imitieren, um sie technisch nutzbar zu machen, führt häufig zu völlig neuen Lösungen, die auf anderem Weg vielleicht nie gefunden worden wären. //

\section{Kontakt \\ Karlsruher Institut für Technologie KIT Karlsruhe apl. Prof. Dr. Hendrik Hölscher Biomimetische Oberflächen hendrik.hoelscher@kit.edu www.imt.kit.edu/hoelscher.php}

